\title{
Genetic deletion of the bacterial sensor NOD2 improves murine Crohn's disease-like ileitis independent of functional dysbiosis
}

\author{
D Corridoni ${ }^{1,2,6}$, A Rodriguez-Palacios ${ }^{1,2,6}$, G Di Stefano ${ }^{1,2}$, L Di Martino ${ }^{1,2}$, DA Antonopoulos ${ }^{3,4}$, \\ EB Chang ${ }^{4}$, KO Arseneau ${ }^{1,2}$, TT Pizarro ${ }^{2,5}$ and F Cominelli ${ }^{1,2,5}$
}

\begin{abstract}
Although genetic polymorphisms in NOD2 (nucleotide-binding oligomerization domain-containing 2) have been associated with the pathogenesis of Crohn's disease (CD), little is known regarding the role of wild-type (WT) NOD2 in the gut. To date, most murine studies addressing the role of WT Nod2 have been conducted using healthy (ileitis/colitis-free) mouse strains. Here, we evaluated the effects of Nod2 deletion in a murine model of spontaneous ileitis, i.e., the SAMP1Yit/Fc (SAMP) strain, which closely resembles CD. Remarkably, Nod2 deletion improved both chronic cobblestone ileitis (by $50 \%$ assessed, as the $\%$ of abnormal mucosa at $24 \mathrm{wks}$ of age), as well as acute dextran sodium sulfate (DSS) colitis. Mechanistically, Th2 cytokine production and Th2-transcription factor activation (i.e., STAT6 phosphorylation) were reduced. Microbiologically, the effects of Nod2 deletion appeared independent of fecal microbiota composition and function, assessed by 16S rRNA and metatranscriptomics. Our findings indicate that pharmacological blockade of NOD2 signaling in humans could improve health in Th2-driven chronic intestinal inflammation.
\end{abstract}

\section{INTRODUCTION}

Nucleotide-binding oligomerization domain-containing 2 (NOD2) is a cytosolic pattern recognition receptor expressed in both hematopoietic and non-hematopoietic cellular compartments. ${ }^{1}$ The primary role of NOD2 is to detect muramyldipeptide, the breakdown product of peptidoglycan, which is present in the cell wall of Gram-positive and Gram-negative bacteria. ${ }^{2}$ On bacterial sensing, NOD2 binds directly to receptor-interacting protein 2 (RIP2) kinase to form the active NOD2:RIP2 signaling complex, which ultimately leads to downstream activation of both nuclear factor (NF)- $\kappa B$ and mitogen-activated protein kinase (MAPK), and subsequent induction of cytokine production. ${ }^{3-5}$ The importance of NOD2 in inflammation was originally highlighted when detailed mapping of chromosome 16 identified polymorphisms in CARD15 (Nod2) as the most frequent genetic alteration associated with Crohn's disease (CD). Specifically, these
CD-associated NOD2 polymorphisms cause loss-of-function, impairing both MAPK and NF- $\kappa \mathrm{B}$ activation and their downstream effects. ${ }^{6-9}$

Although Nod2 variants have gathered much attention, upto $85 \%$ of CD patients have wild-type (WT) Nod2. Either insufficient or overly robust activation of NOD2 signaling has been attributed to downstream deleterious effects that influence the development and progression of $\mathrm{CD} .{ }^{10}$ To date, several studies have examined the role of WT Nod2 in mice, primarily using ileitis-free C57BL/6J knockout (KO) strains exposed to acute forms of chemical-induced colitis (e.g., dextran sodium sulfate (DSS)). The objective of the present study was to determine the effects of WT Nod2 deletion in a spontaneous mouse model of chronic intestinal inflammation (i.e., SAMP1/YitFc (SAMP)), characterized by a progressive cobblestone CD-like ileitis that develops in the absence of chemical, genetic or immunological manipulation. Our group

${ }^{1}$ Department of Medicine, Case Western Reserve University, Cleveland, Ohio, USA. ${ }^{2}$ Case Digestive Health Research Institute, Case Western Reserve University, Cleveland, Ohio, USA. ${ }^{3}$ Biosciences Division, Argonne National Laboratory, Argonne, Illinois, USA. ${ }^{4}$ Department of Medicine, University of Chicago, Chicago, Illinois, USA and ${ }^{5}$ Department of Pathology, Case Western Reserve University, Cleveland, Ohio, USA. Correspondence: F Cominelli (fabio.cominelli@uhhospitals.org).

${ }^{6}$ Co-First Authors.

Received 13 May 2016; accepted 23 September 2016; published online 16 November 2016. doi:10.1038/mi.2016.98 
and others have extensively described this model, demonstrating that SAMP ileitis shows striking similarity to CD, including disease location, histological features, extra-intestinal manifestation and response to therapies effective in treating the human condition. ${ }^{11}$ Importantly, intestinal inflammation progresses to a chronic state that is maintained throughout the lifespans of these mice, and uniquely displays CD-like cobblestone lesions. ${ }^{12}$

Herein, we provide evidence that genetic deletion of Nod2 in SAMP mice decreases the severity of chronic intestinal inflammation, indicating that NOD2 has a proinflammatory role during the chronic phase of CD-like SAMP ileitis and in the development of cobblestone lesions. Using reciprocal bone marrow chimeras of Nod2-deficient $\left(\mathrm{NOD} 2^{-1-}\right.$ ) and WT SAMP mice, we show that NOD2 production is necessary in both the hematopoietic and non-hematopoietic cellular compartments to fully elicit its pro-inflammatory role. Importantly, we demonstrate that Nod2 deletion markedly suppresses classic Th2-type cytokines, supporting the previously published role for Th2 immune predominance during chronic SAMP ileitis. ${ }^{13}$ In addition, SAMP $\times$ NOD2 $2^{-1-}$ mice exposed to DSS (a model of acute colitis/tissue injury) show reduced levels of total, chronic and active colitis, as well as reduced expression of the Th2 cytokine IL-13. Finally, we found that gut dysbiosis does not contribute to this response. Overall, this study shows for the first time that Nod2 deletion improves chronic CD-like ileitis, and suggests that blocking this signaling pathway may be efficacious as a potential therapeutic intervention for established, chronic inflammation in certain subgroups of patients with $\mathrm{CD}$.

\section{RESULTS \\ Nod2 deletion in SAMP mice decreases the severity of chronic ileitis}

Little is known regarding the mechanisms of NOD2 signaling in modulating the chronic phase of spontaneous intestinal inflammation, as most studies have been performed in murine models of chemically-induced acute colitis. ${ }^{14}$ We have previously demonstrated that SAMP mice possess WT Nod2, but have abnormal NOD2 signaling and loss-of-response to muramyl-dipeptide stimulation before the onset of disease. ${ }^{15}$ To test the role of WT Nod2 in chronic SAMP ileitis, we utilized a speed congenic approach to delete Nod2 from the SAMP genomic background $\left(\mathrm{SAMP} \times \mathrm{NOD} 2^{-/-}\right.$, Figure 1a). We then performed time course studies with histological evaluation at early and late stages of disease $(4,10,20-30$ weeks of age), and showed that SAMP $\times$ NOD2 $2^{-1-}$ mice exhibited a significant decrease in chronic ileitis compared with agematched SAMP WT mice (Figure $\mathbf{1 b}$ and $\mathbf{c}$ ). The severity of intestinal inflammation during the chronic phase of disease (20-30 weeks of age) was also assessed by measuring tissue myeloperoxidase (MPO) enzyme activity, and showed a significant reduction compared with SAMP WT mice (Figure 1d). Overall, the loss of Nod2 decreased the histological and biochemical severity of disease, suggesting that NOD2 plays a pro-inflammatory role during chronic ileitis.

\section{Nod2 deletion prevented the progression of cobblestone lesion formation in SAMP mice and reduced the amount of MPO activity within these lesions}

By using three-dimensional-SM assessment and pattern profiling (3D-SMAPgut) to characterize and quantify the percentage of abnormal gut mucosa, ${ }^{12}$ we found potent attenuation of ileal disease severity in SAMP $\times$ NOD2 ${ }^{-1-}$ compared with WT mice, at both the individual (reduced mean $\%$ of abnormal mucosa) and population levels. The latter were characterized by the disappearance of bimodality in disease severity, which is a feature of WT SAMP ileitis in which 50\% of mice are affected with a severe form of the disease, while the remaining had milder disease. Nod2 deletion led to the generation of a population of mice with healthier intestinal $3 \mathrm{D}$ structure and a wider normalized distribution for the percent of abnormal mucosa (Figure 2a-c). Using a stereomicroscopy (SM)-guided sampling strategy, we next determined the biochemical severity of intestinal inflammation in cobblestone lesions compared with normal areas of the ileal mucosa by measuring tissue MPO activity. MPO, primarily located inside cytosolic granules of neutrophils, is a biochemical marker of neutrophilic infiltration and therefore of active inflammation. Thus, areas with abnormal gut mucosa characterized by distortion of villous appearance (cobblestoneinvolved areas) were compared with areas exhibiting normal 3D villous morphology (normal uninvolved areas). Although the extent of biochemical inflammation in areas of normal mucosa (low MPO activity) was comparable for SAMP $\times \mathrm{NOD}^{-1-}$ and WT SAMP mice, involved areas of mucosa exhibited significantly less inflammation (lower MPO activity) in SAMP $\times$ NOD2 $2^{-1-}$ (Figure 2d).

\section{Bone marrow transplantation of SAMP $\times$ NOD2 ${ }^{-1-}$ cells into irradiated SAMP $\times \mathrm{NOD2}^{-1-}$ mice exerted maximal anti-inflammatory effects}

Because NOD2 is expressed in both non-hematopoietic intestinal epithelial cells and hematopoietic antigen presenting cells, ${ }^{16}$ we quantified separately the contribution of NOD2 that is derived from either cellular compartment in the development of chronic intestinal inflammation by generating reciprocal bone marrow (BM) chimeric mice (Supplementary Figure S1 online). Of note, as shown by histologic assessment of ileitis and colitis, SAMP $\times \mathrm{NOD} 2^{-1-} \mathrm{BM} \rightarrow \mathrm{SAMP} \times$ $\mathrm{NOD} 2^{-1-}$ recipients had significantly lower inflammation compared with SAMP WT BM $\rightarrow$ SAMP WT mice, indicating that the anti-inflammatory role of Nod2 deletion could be due to NOD2-dependent signaling mechanisms in both the hematopoietic and non-hematopoietic compartments (Figure 2e). No differences were observed in heterologous transplants $\left(\mathrm{SAMP} \times \mathrm{NOD}^{-1-} \mathrm{BM} \rightarrow \mathrm{SAMP} \quad \mathrm{WT}\right.$ and SAMP WT BM $\rightarrow \mathrm{NOD} 2^{-1-}$ mice), supporting the concept that Nod2 deletion in both compartments exerts the greatest anti-inflammatory effect. 

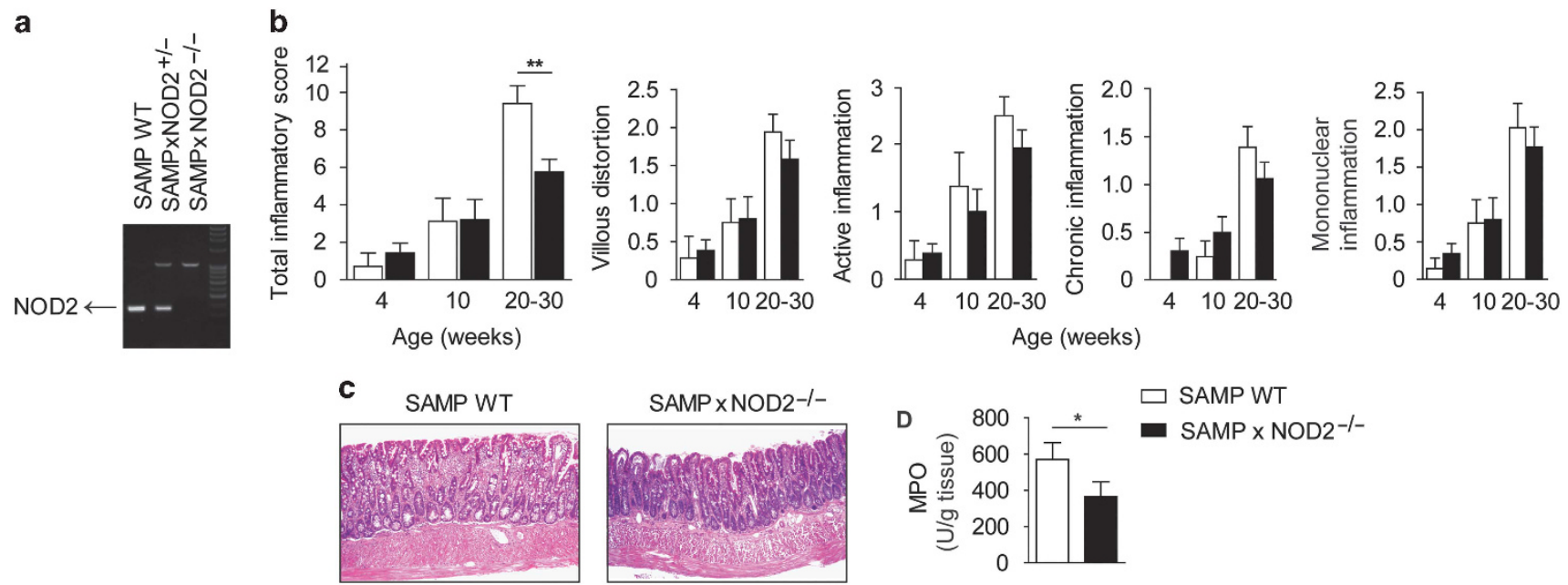

Figure 1 SAMP $\times \mathrm{NOD}^{-1-}$ mice show decreased severity of inflammation compared with SAMP WT mice. (a) PCR genotyping of mouse tail DNA showing the generation of SAMP $\times$ NOD2 ${ }^{-1-}$ mice using speed congenic techniques. (b) lleal total inflammatory scores at different time points $(4,10$ and 20-30-wk-old), as determined by the sum of chronic inflammation, active inflammation, villous distortion and mononuclear inflammation. ${ }^{\star *} P<0.001$ $(n=17-24)$, unpaired Student's $t$ test; data are represented as mean \pm s.e.m. (c) Representative histopathological sections show significant villous blunting and a significant increase of inflammatory cells in the lamina propria of 20-30-wk-old SAMP WT compared with SAMP $\times$ NOD2 ${ }^{-1-}$ mice. (d) MPO activity in ilea of SAMP WT and SAMP $\times$ NOD2 ${ }^{-1-}$ mice at 30 wks of age. ${ }^{*} P<0.05(n=13)$, pairwise Mann-Whitney test; data are represented as mean \pm s.e.m.

\section{Nod2 deletion reduces Th2 cytokine expression during the chronic phase of CD-like ileitis}

Our group has previously shown that both Th1 and Th2 immune responses play an important inflammatory role in the pathogenesis of SAMP ileitis. ${ }^{13}$ During the early inductive phase of intestinal disease in SAMP mice (4-6-wks of age), there is a predominant mucosal Th1 immune response. In contrast, during chronic inflammation in adult mice (20-30wks old), the established Th1 response is coupled with a marked increase in Th2 effector pathways. ${ }^{11,13}$ To explore the effect of Nod2 deletion on the types of immune responses during different stages of ileitis, we measured cytokine secretion from activated mesenteric lymph node (MLN) cells isolated from $\mathrm{SAMP} \times \mathrm{NOD}^{-1-}$ and WT mice during the pre-inflammatory phase at 4 weeks of age, and in the established chronic phase at 30 weeks of age. Nod2 deletion had no significant effect on either Th1 or Th2 cytokines at 4 weeks of age. However, $\mathrm{SAMP} \times \mathrm{NOD}^{-1-}$ mice exhibited a significant decrease in secretion of Th2-type cytokines at 30 weeks of age (i.e., IL-4, IL-5 and IL-13), but not Th1-type cytokines (Figure 3a and b). Altogether, these results strongly indicate that blockade of WT Nod2 decreases Th2-mediated immunity during chronic SAMP ileitis and ameliorates disease severity.

\section{Nod2 deletion modulates Th2 cytokine signaling}

To determine the effects of Nod2 deletion on Th2-dependent cytokine signaling pathways in SAMP mice, we first determined whether NOD2-driven Th2 gene expression varied between normal and abnormal intestinal mucosa in SAMP $\mathrm{WT}$ versus $\mathrm{SAMP} \times \mathrm{NOD} 2^{-1-}$ mice. The expression of GATA-3, a signature master regulator of Th2 activation, ${ }^{17}$ and the production of the Th2 cytokines were measured in
SM-micro-dissected areas from the ilea of SAMP $\times \mathrm{NOD}^{-1-}$ and age-matched WT mice (Figure 4a). Abnormal areas of mucosa from SAMP WT mice exhibited increased expression of GATA-3, IL-4, IL-5 and IL-13 compared with normal areas. In contrast, cytokine expression in abnormal areas of tissue from SAMP $\times \mathrm{NOD}^{-I-}$ was significantly attenuated and comparable to levels expressed in uninvolved mucosal areas (Figure $4 b$ and $c$ ).

To determine if the absence of NOD2 in SAMP mice resulted in the inability to activate Th2-positive regulators during the chronic stage of ileitis, we measured protein levels of phosphorylated-STAT6 compared with total STAT6 in SM-micro-dissected areas of ilea that displayed abnormal mucosa (involved areas) and normal villous morphology (uninvolved area) from 30-wk-old SAMP $\times \mathrm{NOD}^{-1}-$ and age-matched WT mice. Importantly, increased levels of STAT6 phosphorylation were detected in the cobblestone lesions versus normal mucosa of SAMP WT mice, and were not detectable in either tissue type in SAMP $\times \mathrm{NOD}^{-/}$mice (Figure 4d). We next determined the levels of phosphorylatedSTAT6 in MLNs isolated from 30-wk-old SAMP $\times \mathrm{NOD}^{-1-}$ compared with WT mice. High levels of phosphorylatedSTAT6 were detected in SAMP WT MLNs, but not in MLNs from SAMP $\times$ NOD2 ${ }^{-1-}$ mice, whereas levels of total-STAT6 were unchanged between the two groups. Consistently, high protein levels of GATA-3 and IL-13 were also detected in the MLNs of SAMP WT, but not SAMP $\times \mathrm{NOD}^{-/-}$mice (Figure 4e). Collectively, these data provide evidence that NOD2 regulates activation of specific Th2-transcription factors and downstream Th2 cytokines in adult SAMP mice with established inflammatory lesions, thus contributing to the severity of chronic intestinal inflammation. 
a

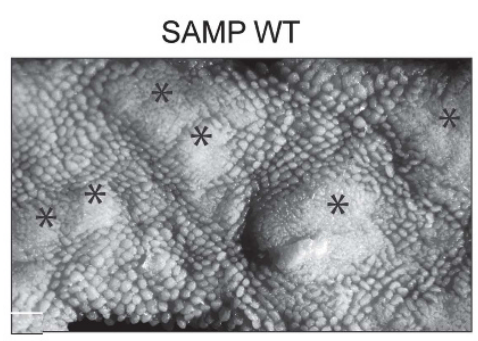

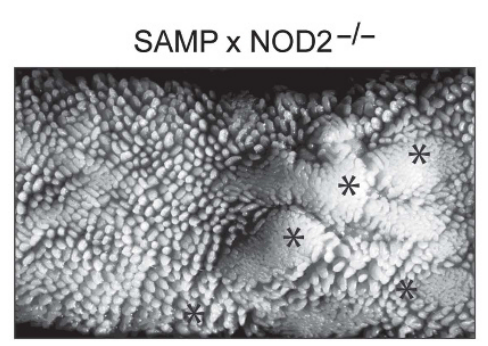

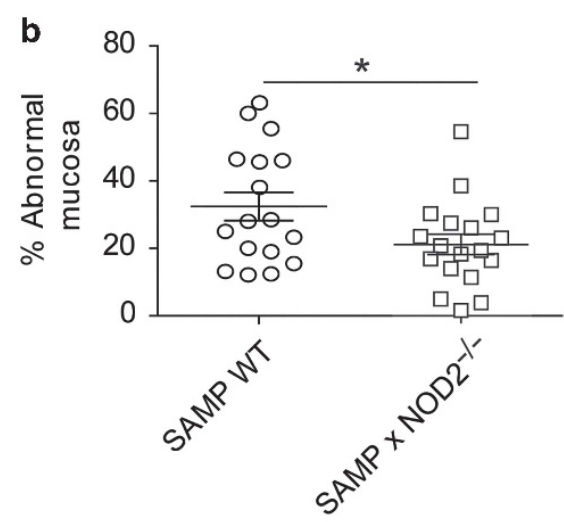

C

\section{SAMP $\times \mathrm{NOD}^{-1-} \quad$ SAMP WT}

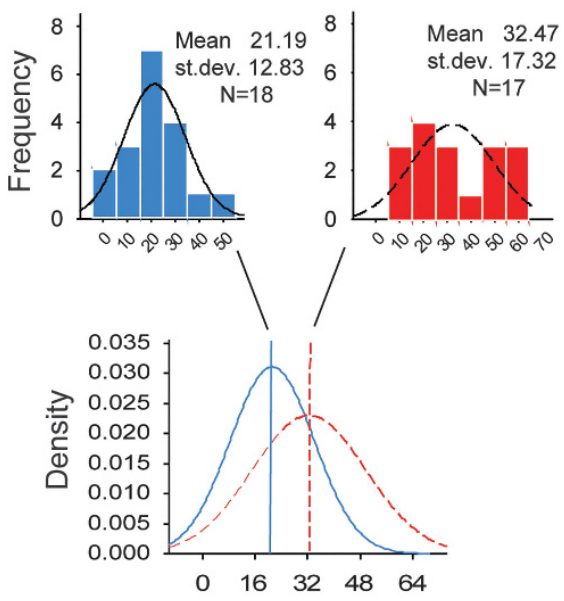

SM \% of abnormal mucosa d

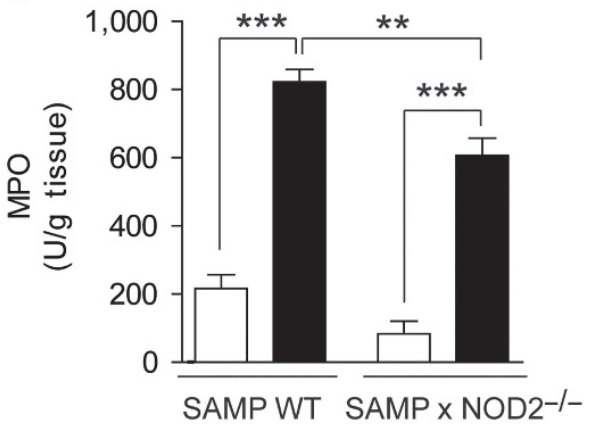

e

SAMP WT SAMP $\times$ NOD2-l-
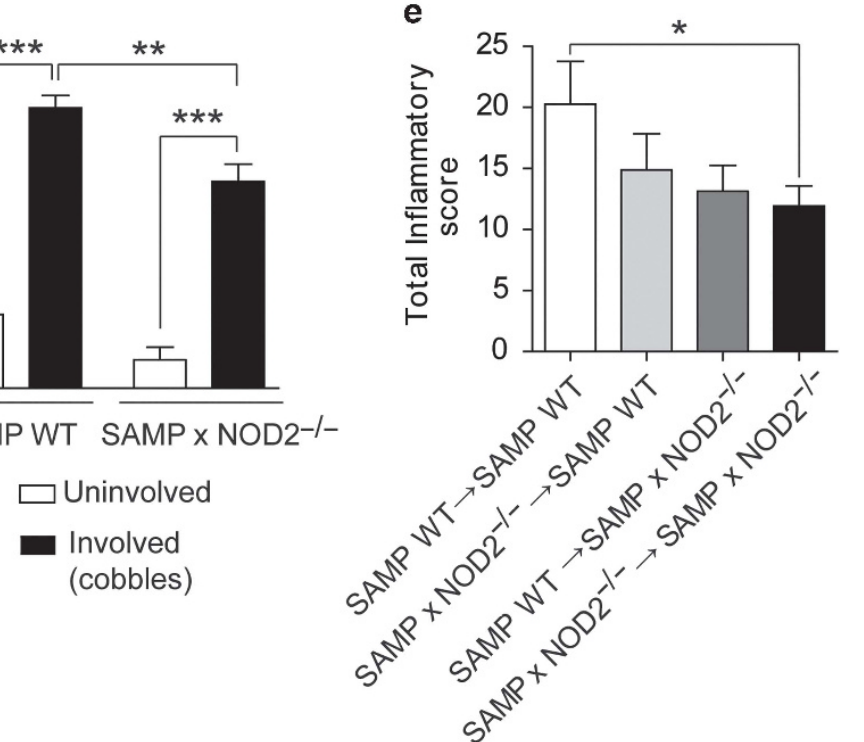

Figure 2 Deletion of Nod2 in SAMP mice decreases the extension of abnormal mucosa. (a) Three-dimensional stereomicroscopic (3D-SM) images of fixed specimens to visualize the architecture of ilea in SAMP WT and SAMP $\times$ NOD2 ${ }^{-1-}$ mice. (b) Percentage of 3D abnormal mucosa in ilea of SAMP WT and SAMP $\times$ NOD2 ${ }^{-1-}$ mice. ${ }^{*} P<0.05(n=17-18)$, Student's $t$ test; data are represented as mean \pm s.e.m. (c) Density plot analysis of data with a bimodal distribution for SAMP WT (with $\sim 50 \%$ of mice affected with severe disease) and normalized (predictable Gaussian) healthy population of SAMP $\times$ NOD2 ${ }^{-1-}$ mice. (d) MPO activity in uninvolved and involved areas of ilea from SAMP WT and SAMP $\times$ NOD2 ${ }^{-1-}$ mice. ${ }^{\star \star} P<0.001$, ${ }^{* \star \star} P<0.0001(n=7)$, pairwise Mann-Whitney test; data are represented as mean \pm s.e.m. (e) lleo-colitis total inflammatory scores of the following groups of chimeric mice: SAMP WT BM $\rightarrow$ SAMP WT, SAMP $\times$ NOD2 ${ }^{-1-}$ BM $\rightarrow$ SAMP WT, SAMP WT BM $\rightarrow$ SAMP $\times$ NOD2 $2^{-1-}$ and SAMP $\times$ NOD2 ${ }^{-1-}$ BM $\rightarrow$ $\mathrm{SAMP} \times \mathrm{NOD}^{-1-} .{ }^{*} P<0.05$. $(n=7)$, Student's $t$ test; data are represented as mean \pm s.e.m.

\section{The anti-inflammatory effects of Nod2 deletion are not attributable to gut dysbiosis}

Recent studies have shown that deletion of Nod2 in healthy C57BL/6J mice plays a proinflammatory role via the induction of proinflammatory and transmissible gut microbiota perturbation. ${ }^{18}$ Because the SAMP ileitis is progressive and spontaneous with $100 \%$ penetrance, we determined whether the NOD2-driven, Th2-mediated proinflammatory effects in chronic stages of SAMP ileitis were dependent on gut microbiota changes within the intestinal tract. Given that the mouse gut microbiome quickly responds to dietary changes within $24 \mathrm{~h}$ of feeding manipulation, we quantified the extent of compositional and functional divergence in the gut microbiomes of 6 -wk old SAMP WT and SAMP $\times \mathrm{NOD}^{-1-}$ mice (i.e., during the induction phase of disease, when the percent of abnormal mucosa is negligible). Seven days after the inter-subject pre-experimental fecal homogenization (IsPreFeH protocol, Supplementary Figure S2), ${ }^{19}$ fecal samples were collected from each individually-caged mouse for $16 \mathrm{~S}$ rRNA-based microbiome analysis of the gut microbial community, and functional and metabolic metatranscriptome analysis. Interestingly, no acute fecal microbiota community or functional changes (dysbiosis) were detected in 6-wk old $\mathrm{SAMP} \times \mathrm{NOD} 2^{-1-}$ mice compared with WT mice, consistent with our findings that $\operatorname{Nod} 2$ deletion did not affect ileitis severity or Th1 and Th2 cytokine production during this early, inductive stage of SAMP ileitis. Metabolically, no dysbiosis was detected at the family-level between groups based on metatranscriptome profiles, indicating that the community composition of the fecal microbiota is functionally similar between WT and SAMP $\times$ NOD2 ${ }^{-1-}$ mice (Figure 5a and $\mathbf{b}$ ). MG-RAST visualization of normalized transcript functional 

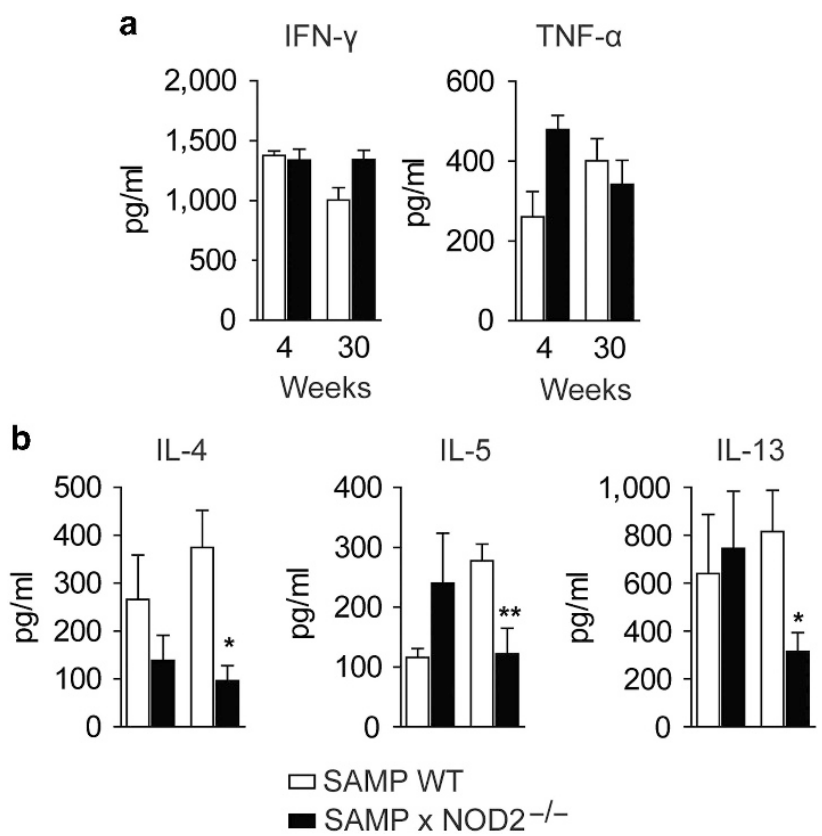

Figure 3 Decreased production of Th2 cytokines from MLN cells of $\mathrm{SAMP} \times \mathrm{NOD} 2^{-1-}$ mice. Secretion levels of Th1-type cytokines (a), TNF$\alpha$ and IFN- $\gamma$, and Th2-type cytokines (b), IL-4, IL-5 and IL-13 from MLN $\mathrm{CD}^{+}{ }^{+} \mathrm{T}$ cells isolated from pre-inflamed (4-wk old) and inflamed (30-wkold) SAMP WT and SAMP $\times$ NOD2 ${ }^{-1-}$, following polyclonal activation with anti-CD3/anti-CD28. ${ }^{\star} P<0.05,{ }^{*} P<0.01(n=6-10)$, Mann-Whitney test; data are represented as mean \pm s.e.m.

abundance (\%) from the 6 individual metatranscriptomes illustrated that no major differences in metabolism were evident (subsystem levels 1 and 2), including microbial virulence markers, to explain differences in disease severity between $\mathrm{WT}$ and SAMP $\times \mathrm{NOD} 2^{-1-}$ mice (Figure 5c). Analysis of the metatranscriptomes revealed a trend in the 50 most expressed functional roles (specific enzymatic reactions within the subsystems framework) detected between SAMP WT and SAMP $\times \mathrm{NOD} 2^{-1-}$ mice; however, none exhibited significant differences in their fold-changes (Figure 5d). Agglomeration of the 7,473 functional roles detected at a higher-level classification (subsystems level 2) revealed differences $(P<0.05)$ in the log-fold changes between groups for only a minimal fraction $(n=4 ; 2.07 \%)$ of 193 level 2 categories identified, indicating that $\mathrm{Nod} 2$ deletion does not change the overall metabolic role of the gut microbiota. An example of the magnitude of functional changes (e.g., sugar phosphotransferase systems) is depicted in Figure 5e.

No metatranscriptome-based studies were conducted in advanced stages of disease (e.g., 30-wk-old mice) since the genetic deletion inherently results in less inflammatory changes in SAMP $\times \mathrm{NOD} 2^{-1-}$ mice, which would have confounded interpretation of any outcome derived from studies comparing inflamed and less-inflamed mice. However, because our microbial studies supported the concept that the gut microbiota remains stable for at least 7 days following fecal homogenization, we used the same protocol to assess susceptibility to DSS- colitis in young mice in order to determine if Nod 2 deletion also protects SAMP mice from chemically-induced acute intestinal injury.

\section{Nod2 deletion minimizes the severity of DSS colitis in young SAMP mice}

On the basis of observation that no significant changes were found in the composition or function of the gut microbiota in young mice, we then determined how NOD2 deficiency intrinsically modulates the risk of disease using a chemicallyinduced model of colitis. Following 7 days of exposure to a composite mixture of feces and bedding material from both WT and SAMP $\times \mathrm{NOD}^{-1-}$ mice (IsPreFeH protocol, Supplementary Figure S2), animals were exposed to 3\% DSS in drinking water for 7 days. As shown in Figure 6a, DSS-treated $\mathrm{SAMP} \times \mathrm{NOD} 2^{-1-}$ mice lost significantly less body weight compared with WT mice, and had less evidence of mucosal colonic lesions as assessed by endoscopy (Figure $\mathbf{6 b}$ and $\mathbf{c}$ ). $\mathrm{SAMP} \times \mathrm{NOD} 2^{-1-}$ mice displayed milder inflammation, slight vascular changes, and granularity, whereas SAMP WT mice showed thickened mucosa, irregular vascular patterns, and a higher number of ulcers. Similarly, histology and MPO activity showed SAMP $\times \mathrm{NOD} 2^{-1-}$ mice were less likely to develop severe DSS colitis compared with WT mice (Figure 6d-f).

In the same experiment, we also measured colonic expression of IL-13, a prototypic Th2 cytokine, to determine if the NOD2-driven Th2 cytokine induced in spontaneous chronic SAMP ileitis also occurred in DSS-induced colitis. As shown in Figure 6g, colonic IL-13 expression was significantly decreased in SAMP $\times \mathrm{NOD} 2^{-1-}$ compared with WT mice. By comparison, no differences were detected in colonic TNF- $\alpha$ expression (a prototypic Th1 cytokine) in WT or $\mathrm{SAMP} \times \mathrm{NOD} 2^{-1-}$ mice (Supplementary Figure S3). Altogether, these data confirm that NOD2 plays a proinflammatory role during acute intestinal injury caused by DSS-induced colitis in SAMP mice by modulating Th2-type immune responses, an effect that cannot be attributed to changes in the gut flora.

\section{Microbiome 16S composition between SAMP $\times$ NOD2 ${ }^{-1-}$ and SAMP WT mice cannot predict the Nod2 deletion}

To directly determine whether Nod2 deletion diminishes the severity of DSS-induced colitis independent of dysbiosis, we conducted two additional DSS experiments to evaluate the $16 \mathrm{~S}$ microbiome composition before and after DSS administration. Pooled statistical analysis of the $16 \mathrm{~S}$ data, controlling for experimental replica, indicated that the fecal microbiome differences between SAMP $\times$ NOD2 $2^{-1-}$ and WT mice were negligible and could not predict the Nod 2 deletion using logistic regression despite the microbiome differences attributed to DSS-induced colitis (Figure 7a). These data provide further evidence that the anti-inflammatory effects of Nod2 deletion in DSS colitis are not statistically associated with a geneticallydriven intestinal dysbiosis pattern (logistic regression model; $P>0.1 ;$ Nod 2 deletion as outcome; bacterial phyla as dependent variables). Principal coordinate analysis illustrated that 

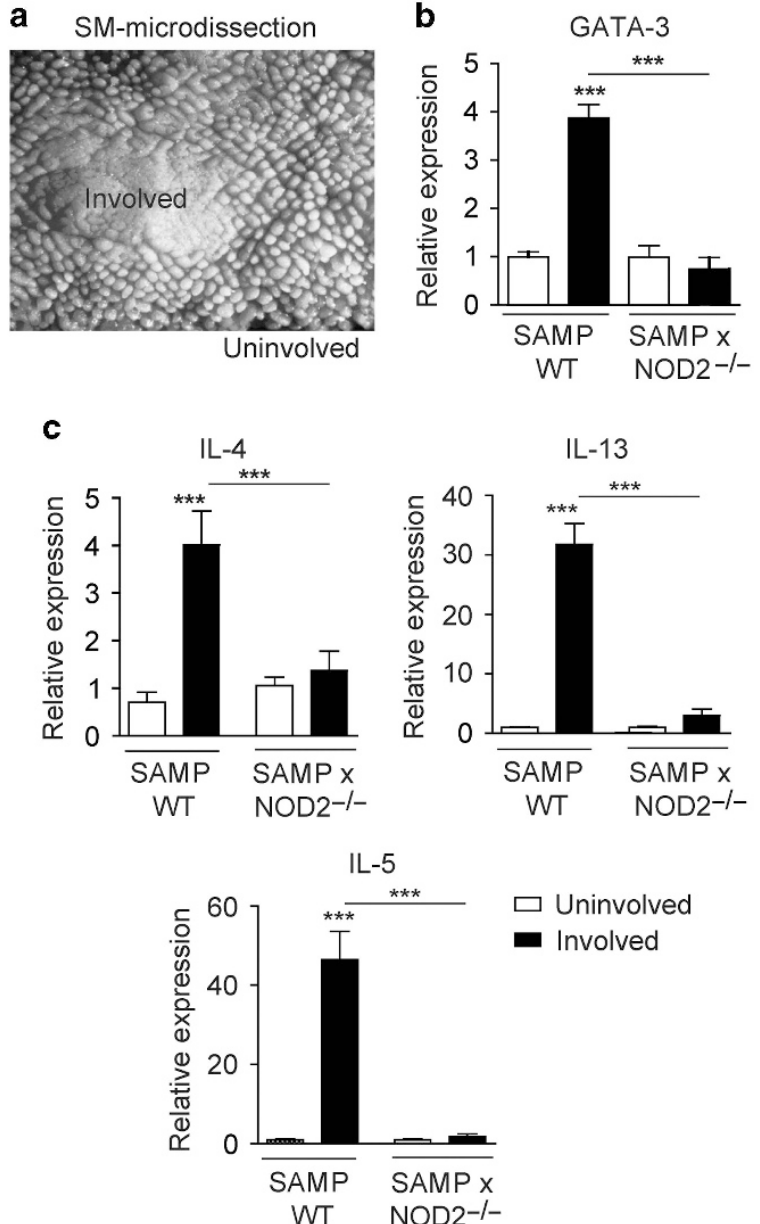

d

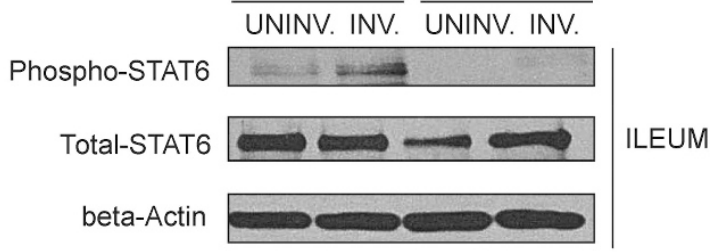

e

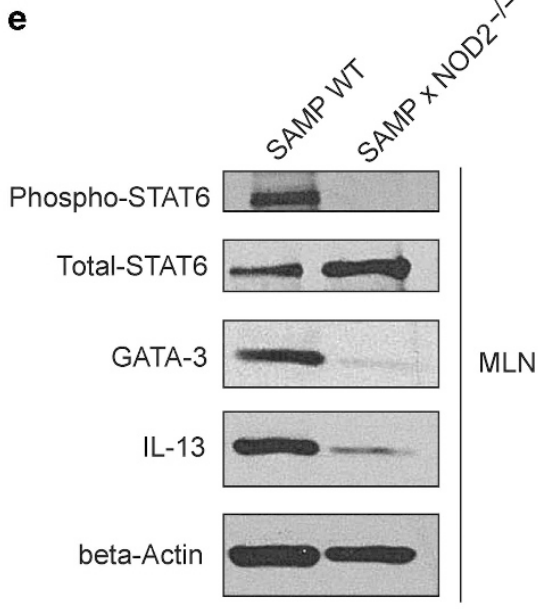

Figure 4 NOD2 regulates Th2-mediated chronic intestinal inflammation. (a) Representative 3D-SM image of involved and uninvolved areas of SAMP WT and SAMP $\times$ NOD2 ${ }^{-1-}$ mice from which samples were derived. (b) GATA-3, (c) IL-4, IL-5 and IL-13 expression during chronic ileitis (30 wks). ${ }^{* \star *} P<0.0001(n=6-12)$, Student's $t$ test; data are represented as mean \pm s.e.m. Protein lysates from (d) ilea and (e) MLNs from 30 -wk-old SAMP WT and SAMP $\times$ NOD2 $^{-1-}$ mice immunoblotted with antibodies against phosphorylated-STAT6, total-STAT6, GATA-3, IL-13 and actin ( $n>3$ ).

$\mathrm{SAMP} \times \mathrm{NOD} 2^{-1-}$ and WT mice cluster together, with most of the community composition differences attributable to the induction of DSS colitis, and not to Nod2 deletion (Figure 7a and $\mathbf{b}$; Figure 8).

Altogether, these results support the concept that the clinical effect of Nod2 deletion on intestinal severity, at least in young SAMP mice and during the acute phase of intestinal inflammation, occurs in a manner that cannot be attributed to changes in the gut microbiota, either naturally due to the gene deficiency or inducibly due to DSS administration.

\section{DISCUSSION}

In the present study, we demonstrate that deletion of WT Nod2 markedly ameliorates chronic CD-like ileitis in SAMP mice, with $\sim 50 \%$ mucosal damage prevention by 24 wks of age. We also determine that the anti-inflammatory effects of Nod2 deletion involve suppression of Th2-type immune responses, previously hypothesized to be an important mediator of disease during the chronic stage of SAMP ileitis. In addition, we showed that Nod2 deletion does not result in acute dysbiotic changes in SAMP mice, indicating that NOD2 itself is a driving force behind Th2-mediated chronic SAMP ileitis, independent of gut microbial composition (Figure 8).

In support of the clinical relevance of these findings, there is evidence that some CD patients display overexpression and hyperactivity of small intestinal NOD2 signaling, as well as its obligate kinase, RIP2, in the presence of WT NOD $2 .{ }^{20}$ As such, it has been suggested that increased expression of WT NOD2 may represent a mechanism by which inflammation is intensified in humans that are prone to $\mathrm{CD} .{ }^{16}$ In mice, previous studies of Nod 2 deletion, primarily using mice on an intestinal disease-free genetic background (e.g., C57BL/6J), have shown complementary results. Nod2 deletion ameliorates the severity of intestinal inflammation in IL-10 $0^{-1-}$ mice, a model in which the development of colitis is dependent on gut microbiota composition. ${ }^{21}$ In the present study, using an ileitisprone mouse model, we found that Nod2 deletion significantly attenuated the severity of spontaneous disease in adult SAMP mice. In accordance with the central role played by NOD2 in orchestrating innate inflammatory responses and protecting 


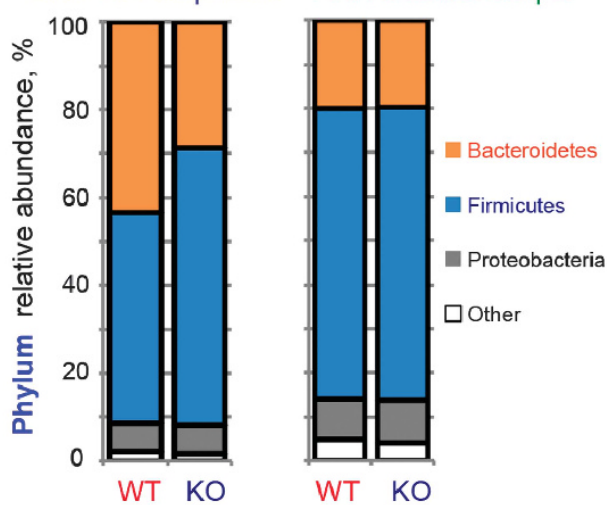

C

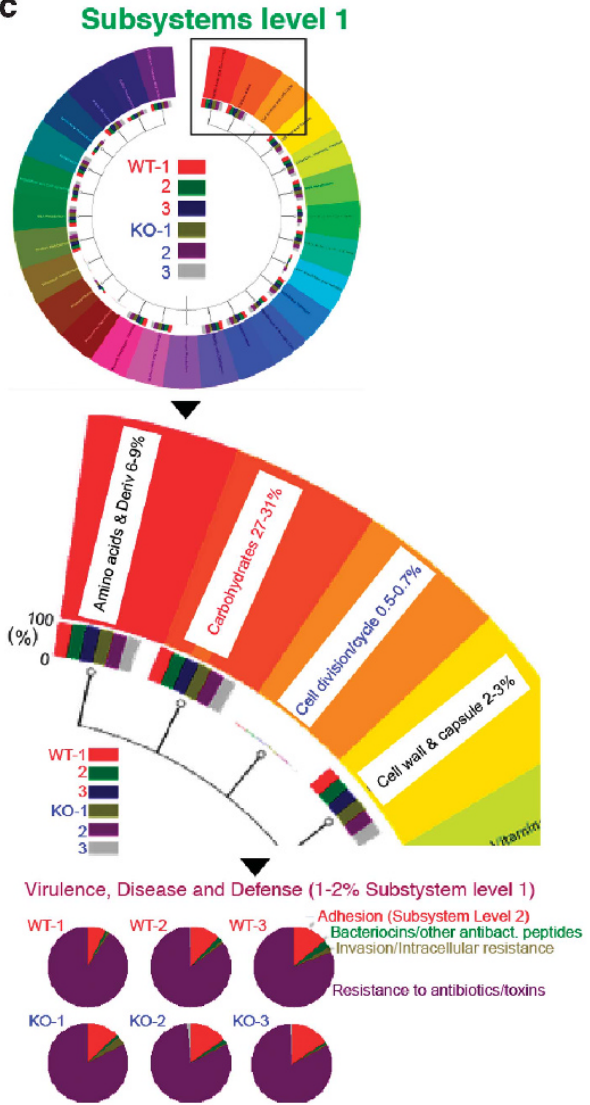

b

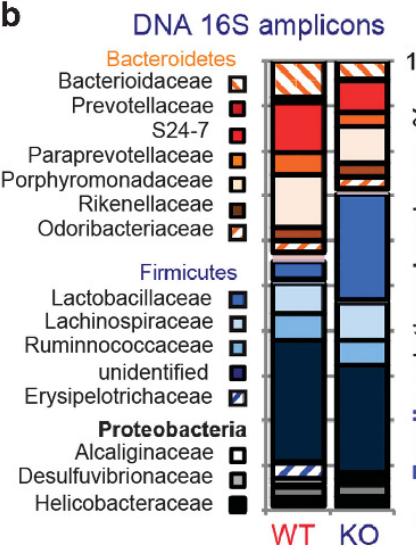

RNA metatranscripts

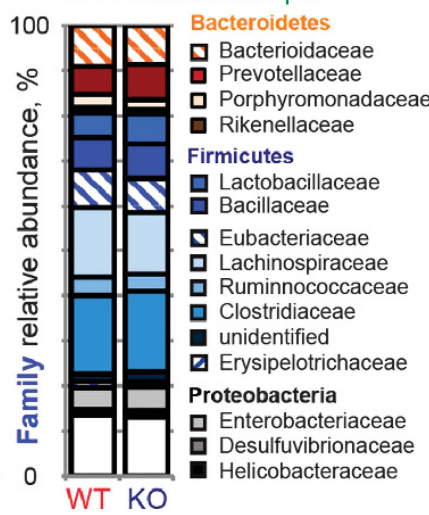

d

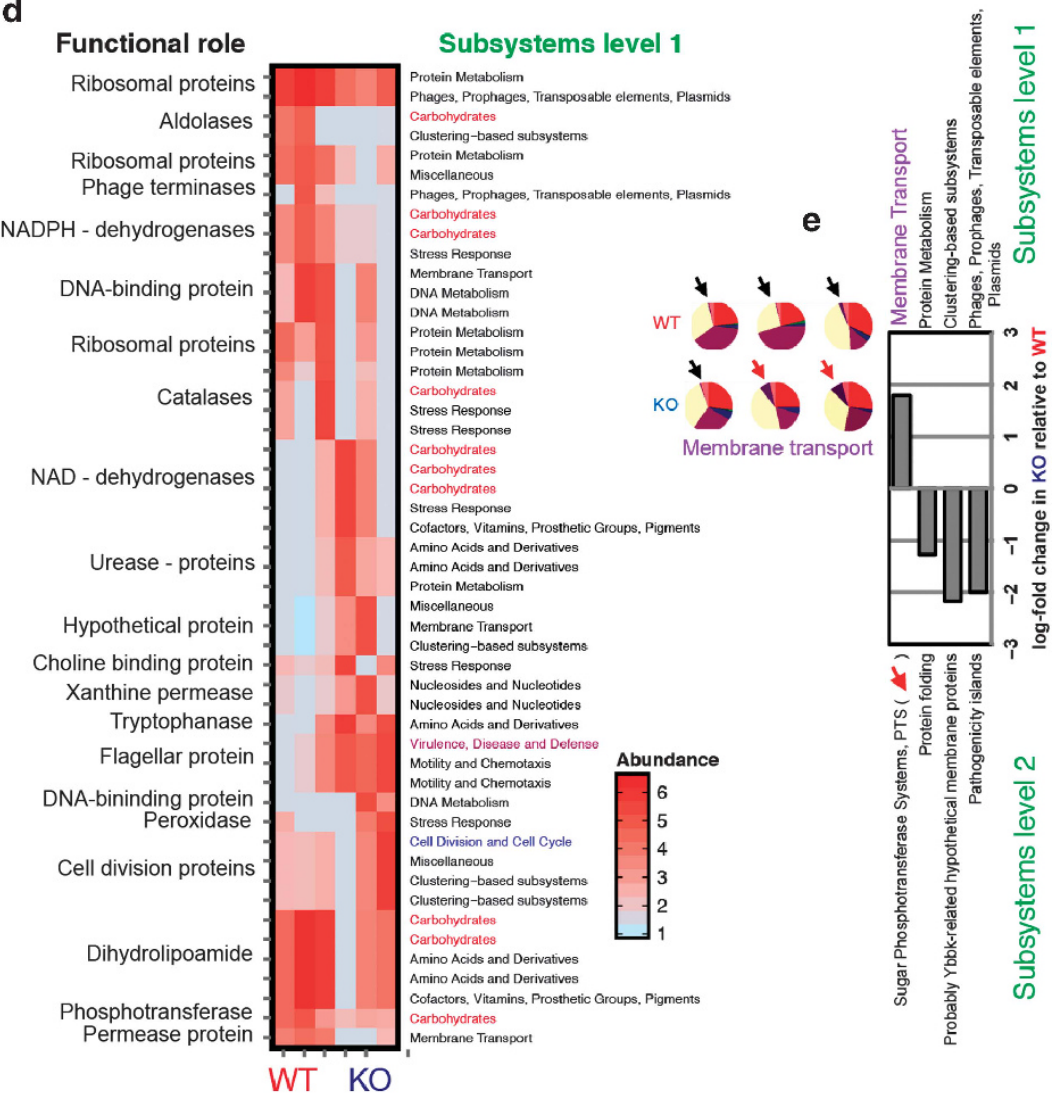

Figure 5 Gut metatranscriptome analysis indicates the Nod2 deletion did not have a significant impact on fecal microbiota composition and function. Analysis on samples collected 7 days following IsPreFeH (3 mice per group). (a) Phylum-level composition based on 16S rRNA amplicon libraries and phylogenomic taxonomic inference from functional mRNA metatranscriptome data. (b) Family-level composition based on 16S rRNA amplicon libraries and phylogenomic taxonomic inference from functional mRNA metatranscriptome data (c) MG-RAST visualization of normalized transcript functional abundance (\%) from the 6 individual metatranscriptomes. (d) Metatranscriptome cluster analysis of most expressed roles (specific enzymatic reactions). (e) Log-fold changes for the only 4 significantly detected functional roles ( 4 out of 193 level 2 categories). Pie charts, relative metatranscript abundance for the sugar phosphotransferase system.

the intestinal mucosa under normal physiological conditions, we propose that WT NOD2, on a human genetic background that could predispose to $\mathrm{CD}$, may have dichotomous roles such that any deviation, either positive or negative, may cause immunological dysfunction promoting chronic intestinal inflammation. Of note, this dichotomous role has been described, not only for NOD2 signaling, but also for other important proinflammatory cytokines, such as TNF, IL-1, IL-18, and IL-33, as well as important transcription factors activated by NOD2 signaling, such as a NF- $\kappa \mathrm{B} .{ }^{22}$

Our results show that different components of the mucosal immune system's signaling pathways are affected by the absence of NOD2. In fact, we demonstrated that Nod2 deletion was associated with inhibition of the Th2 cytokines IL-4, IL-5 

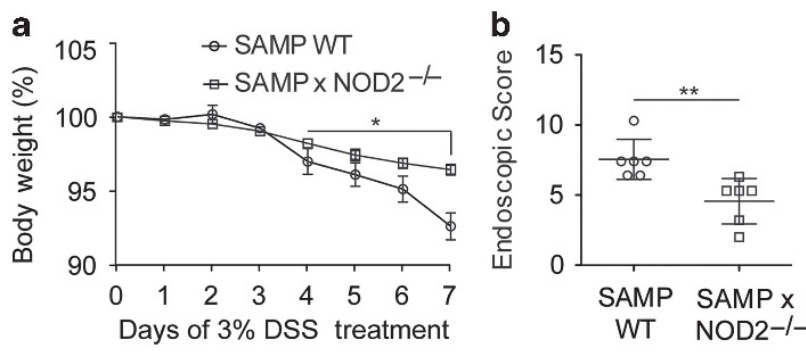

c
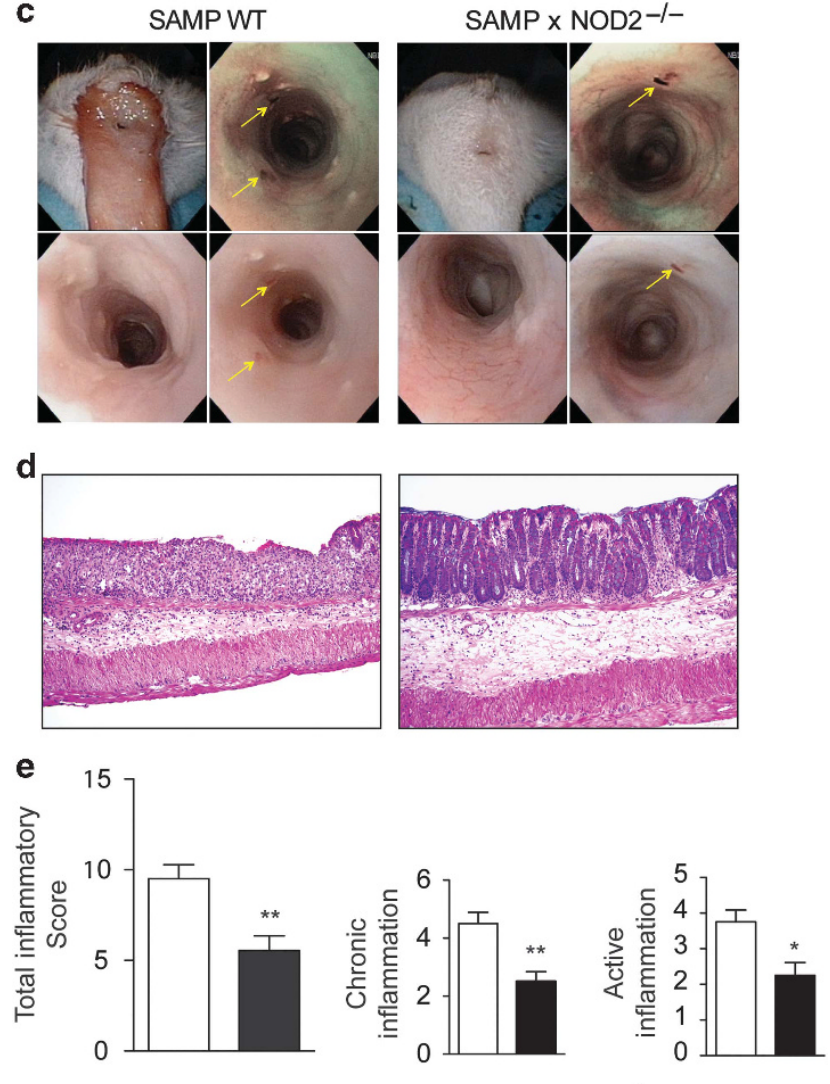

$\square$ SAMP WT
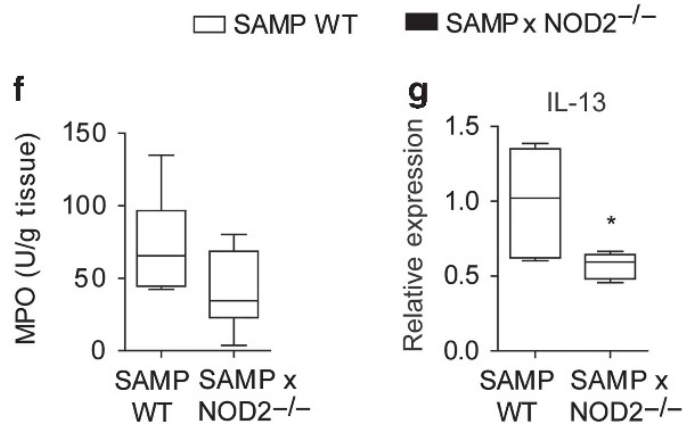

and IL-13, which are important in fibrosis, eosinophilia and Th2 immunity, ${ }^{23}$ whereas no effect was observed in Th1 cytokine expression, including TNF- $\alpha$ and IFN- $\gamma$. Recent studies have shown that neutrophils can be associated with type 2 immunity and Th2 immune responses. ${ }^{24}$ In addition, eosinophils have been shown to play an important role in type 2 immune responses and pathologic conditions, including allergy, asthma and Crohn's disease. ${ }^{25}$ In regards to the role of
Figure 6 Following IsPreFeH, SAMP $\times$ NOD2 ${ }^{-1-}$ mice show reduced severity of DSS colitis compared with WT mice. After 4 days of exposure to a composite mixture of feces and bedding material (orally gavaged), SAMP WT and NOD2 ${ }^{-1-}$ mice were treated with $3 \%$ DSS in their drinking water for 7 days ( $n=6 /$ group). (a) Changes in body weight during DSS treatment (AUC, Student's $t$ test $P<0.05$ ). (b) Endoscopic score based on a distal-proximal endoscopic colitis scoring system on day 7. (MannWhitney, ${ }^{* *} P<0.01$ ); data are represented as mean \pm s.e.m. (c) Endoscopic images of perianal, distal and proximal colon after DSS treatment show severe inflammation in SAMP WT mice (thickened appearance of the mucosa and numerous ulcers-yellow arrows) compared with mild inflammation in SAMP $\times$ NOD2 ${ }^{-1-}$ mice. (d) Representative histopathology images at same magnification show thickening and increased cellular infiltration in lamina propria of SAMP WT mice. (e) Histological, total, chronic and active colonic inflammation scores (Mann-Whitney, ${ }^{* *} P<0.01,{ }^{*} P<0.05$ ). (f) MPO activity in colonic tissue after DSS treatment. (g) IL-13 in colonic tissue after DSS treatment (Mann-Whitney, ${ }^{*} P<0.05$ ); data are represented as mean \pm s.e.m.

PMNs in chronic ileitis, the role of both neutrophils and eosinophils in this condition remains controversial. While both cell types have been shown to play a protective role in certain mouse models of intestinal inflammation (primarily DSS colitis), it is clear that in conditions of chronic gut inflammation, both neutrophils and eosinophils can play a pathogenic role. ${ }^{26}$ The role Th2 effector signaling pathways were also affected by NOD2 deletion, with decreased phosphorylatedSTAT6 and GATA3 in the gut mucosa of SAMP $\times$ NOD2 ${ }^{-1-}$ mice. Consistently, we found that NOD2 requires both hematopoietic and non-hematopoietic compartments to fully mediate its anti-inflammatory effects within the intestinal mucosa, as demonstrated by bone marrow chimera experiments. These results are in line with other studies showing that direct triggering of NOD2 by its ligand, muramyl-dipeptide, generates a specific Th2-type immune response, and that NOD2 expression within the stromal compartment is necessary for priming of effector $\mathrm{CD} 4{ }^{+} \mathrm{Th} 2$ responses and sensing of muramyl-dipeptide by dendritic cells, but not sufficient to induce Th2 immunity. ${ }^{4,27}$ However, the detailed mechanisms that underlie the beneficial effects of NOD2 blockade in intestinal health remain unclear. It is possible that NOD2 blockade or suppression acts synergistically with other components of the innate immune system, such as other pattern recognition receptors, to diminish inflammatory responses against intestinal microorganisms in a manner resembling that which is seen in SAMP mice raised under germfree conditions, which have dramatically reduced disease severity in adulthood. ${ }^{28}$

Several studies have reported that NOD2 deficiency causes dysbiosis of the gut microbiota, which in turn has been linked to CD. ${ }^{29,30}$ A recent fecal material transfer study in mice based on 16S microbiome analysis showed that Nod2 deletion in otherwise healthy C57BL/6J mice increased the severity of DSS-induced colitis by inducing a particular dysbiosis within the gut microbiota, which appeared to cause disease in recipient mice after transplantation with dysbiotic feces (i.e., transmissible DSS-colitis risk). ${ }^{18}$ In our study, we demonstrated that the effects of Nod2 deletion in SAMP mice had no significant effect 
a

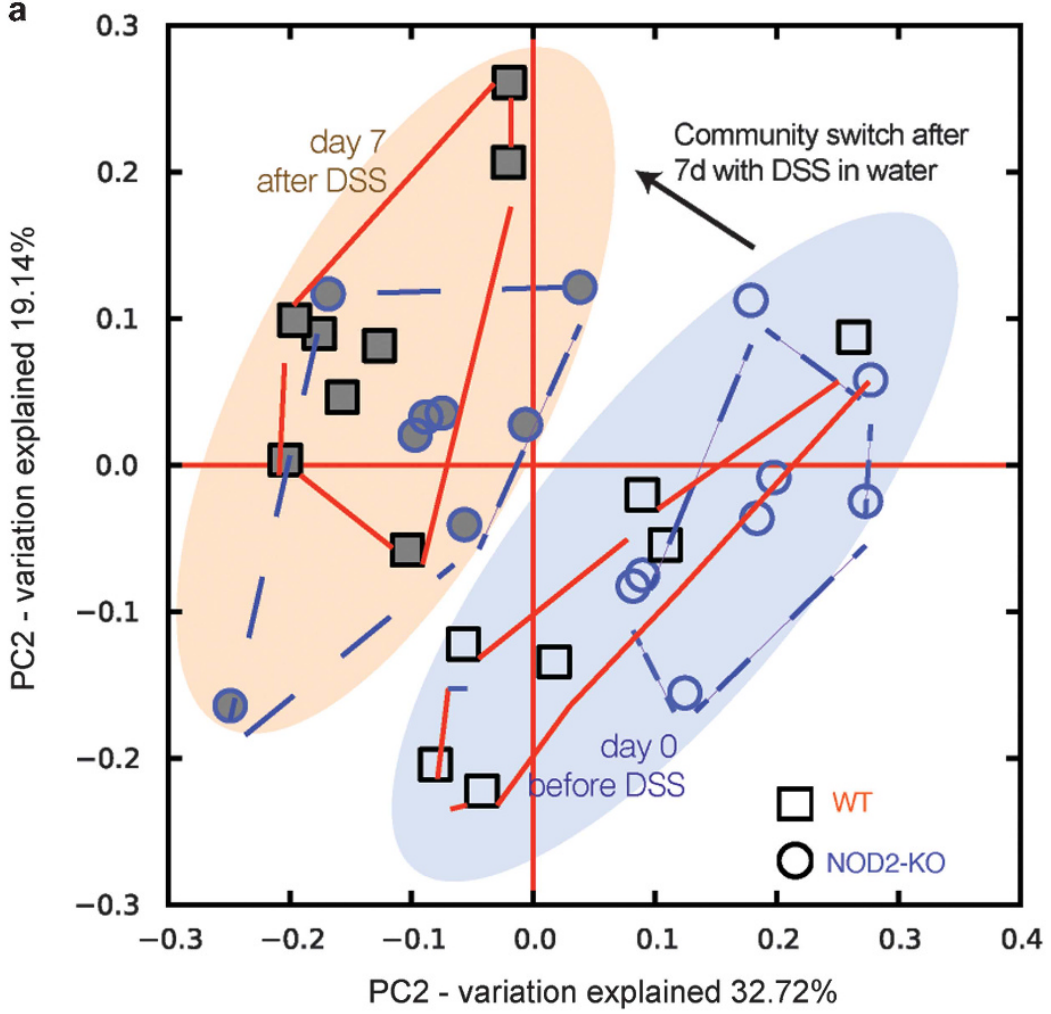

b

day 0 (before DSS)_day 7 (after DSS)

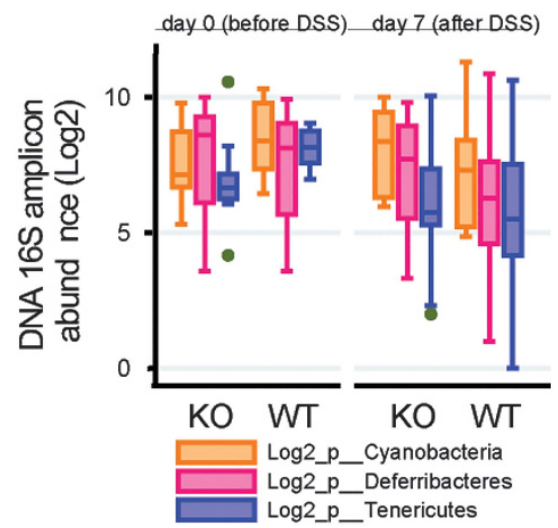

Figure 7 16S rRNA microbial community analysis shows that bacterial community changes before and after DSS-induced colitis do not predict the presence of Nod2 deletion in SAMP mice. Following the fecal homogenization protocol, fecal samples from SAMP WT and SAMP $\times$ NOD2 ${ }^{-1-}$ mice were collected before and after 7 days of $3 \%$ DSS treatment ( $n=3-6 /$ group, 2-3 experiments). (a) Principal coordinate analysis of $16 S$ rRNA microbial community data before and after DSS. (b) Box plot of the normalized 16S amplicon Log2 abundance for the six most abundant bacterial phyla for all experimental mice.

on the microbial composition or bacterial transcriptomic functionality of the fecal flora by analyzing $>1.57$ billion metatranscriptomic illumina sequence reads. Most importantly, we illustrated that by using the same samples and $16 \mathrm{~S}$ microbiome analysis, the microbiota was different. Because the cost of metatranscriptome analysis is prohibitive, we performed and pooled 16S data from a total of three independent DSS colitis experiments, following our IsPreFeH protocol (as an alternative to mouse co-housing) to determine if the $16 \mathrm{~S}$ microbiome profile would be reproducible and predictive of Nod2 deletion in SAMP mice. Of relevance, logistic regression of the pooled data, controlling for all variables, mouse individuality and experiment replica, showed that no single microbial phyla could predict the presence or absence of Nod2 deletion. These data support the findings of the metatranscriptomic analysis, showing that no community changes or clinical effects of intestinal inflammation can be induced by microbiota changes attributable to Nod2 deletion. Our results are consistent with a recent report showing that Nod2 deficiency does not alter the composition of intestinal bacterial communities in homeostatic states, when controlling for housing conditions. ${ }^{31}$

In conclusion, compositional analysis of the microbial community and its function in $\mathrm{SAMP} \times \mathrm{NOD} 2^{-1-}$ and 


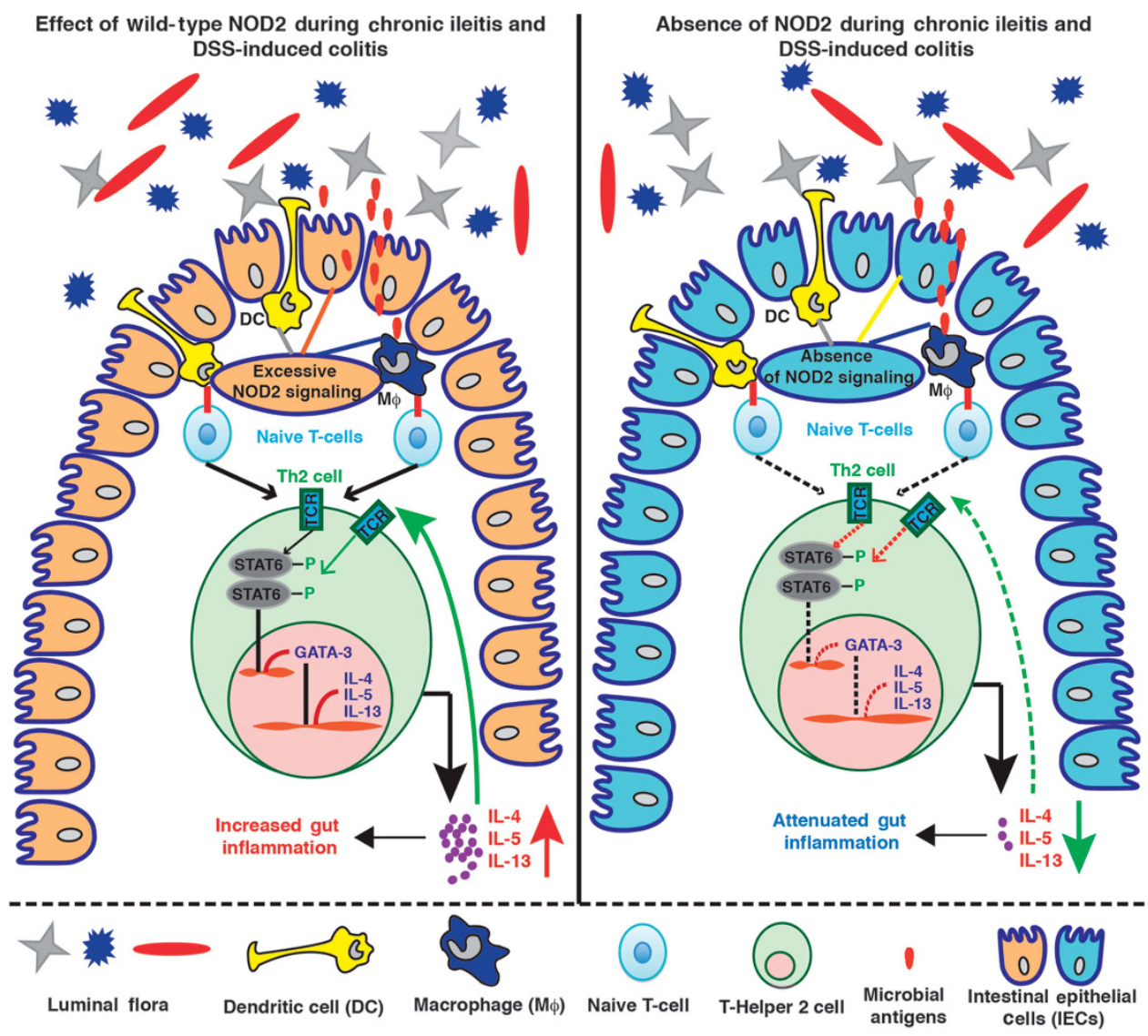

Figure 8 Working hypothesis for the role of NOD2 signaling in experimental IBD. In the presence of WT NOD2 signaling, SAMP mice with spontaneous chronic ileitis or DSS-induced colitis show increased production of Th2 cytokines, such as IL-4, IL-5 and IL-13, which is mediated by excessive NOD2 signaling (left panel). In the absence of NOD2 signaling following NOD2 genetic deletion, there is inhibition of Th2 cytokine production with attenuated gut inflammation (right panel). Mechanistically, these effects are mediated by the activity of Th2-transcription factors, STAT6 and GATA-3 (left and right panel).

WT mice revealed that NOD2 does not have an acute impact on the metatranscriptome composition or function of the commensal flora. This was confirmed by the observation that $\mathrm{SAMP} \times \mathrm{NOD} 2^{-1-}$ and WT mice, following exposure to an identical fecal composite, exhibited different susceptibility to DSS-induced colitis. The fact that SAMP $\times \mathrm{NOD}^{-1-}$ mice showed decreased severity of inflammation compared with WT mice in the presence of the same bacterial flora strongly suggests that changes in the bacterial community are not associated with the effects of NOD2 in ameliorating intestinal inflammation in this model, and that NOD2 regulates Th2 responses in the intestine independent of acute dysbiosis. To further test the efficacy of suppressing NOD2 in treating $C D$, we are currently conducting experiments to describe in greater detail the mechanisms specifically linked to the clinical benefits observed in SAMP mice, and ultimately to translate these findings to humans carrying the WT NOD2 gene, in the total absence of gut commensal microorganisms. These investigations are particularly relevant since we previously documented that Th2 effector pathways require the presence of the gut microbiota. ${ }^{28}$ In fact, compared with specific pathogen-free (SPF) SAMP mice, ileitis in germ-free SAMP is attenuated and associated with delayed lymphocytic infiltration and reduced expression of Th2 cytokines. However, the results of the current study clearly demonstrate that NOD2 regulates Th2 immune responses in the gut, independent of acute changes in the composition or function of the gut microbiome. With the availability of new drug compounds that target inhibition of NOD2 and RIP2-signaling, ${ }^{32-34}$ the present work indicates that potential pharmacologic inhibition of NOD2 signaling may be a reasonable therapeutic strategy to prevent Th2-driven intestinal inflammation, at least in a sub-group of patients with $\mathrm{CD}$.

\section{METHODS}

Experimental animals. SAMP1/YitFc (SAMP) mice were propagated at Case Western Reserve University, with founders obtained from the University of Virginia. A marker-assisted selection protocol was used to generate congenic SAMP mice carrying deletion of the Nod2 gene. SAMP WT littermates were used as controls. SAMP WT and $\mathrm{SAMP} \times \mathrm{NOD} 2^{-1-}$ mice were maintained under SPF conditions, fed standard chow (Harlan Teklad, Indianapolis, IN, USA), and kept on 12-h light/dark cycles.

Induction of DSS colitis. DSS colitis was induced as previously reported. ${ }^{15}$ Briefly, sterilized 3\% (w/v) DSS was added to the drinking 
water of mice for 7 days. Adult SAMP $\times \mathrm{NOD}^{-1-}$ and SAMP WT mice were allowed ad libitum access to water. Daily monitoring was performed for body weight, fecal bleeding and the presence of loose stools.

Histology. Ilea and colons from SAMP WT mice and SAMP $\times$ NOD2 ${ }^{-1}$ mice were removed, flushed of fecal contents, opened longitudinally and placed in Bouin's fixative. Tissues were embedded in paraffin and stained with hematoxylin and eosin. Inflammation was evaluated by a trained pathologist in a blinded fashion using a previously described scoring system. ${ }^{15}$ The total inflammatory score for ileal specimens was the sum of four individual indices for active and chronic inflammation, villous distortion and mononuclear inflammation. The colon total inflammatory score represented the sum of sub-indices for active and chronic inflammation, percent re-epithelialization and percent ulceration.

Stereomicroscopic 3D-pattern profiling of intestinal inflammation. 3D-SM Assessment and Pattern Profiling (3D-SMAPgut) protocol was used to map and quantify the intestinal health in SAMP mice and to determine the extent of mucosal involvement that occurs with acute and chronic inflammation as previously described. ${ }^{12}$

Cytokine protein measurements. IFN- $\gamma$, TNF- $\alpha$, IL-4, IL- 5 and IL-13 were measured by ELISA (eBioscience, San Diego, CA, USA) from MLN cell supernatants following 72-h stimulation with coated with anti-mouse CD3 $\left(1 \mu \mathrm{g} \mathrm{ml}^{-1}\right)$ and soluble anti-CD28 $\left(0.5 \mu \mathrm{g} \mathrm{ml}^{-1}\right)$ (BD Bioscience, San Jose, CA, USA).

MPO assay activity. Miniature specimens $(10 \pm 6 \mathrm{mg})$ were obtained by SM-microdissection according to $3 \mathrm{D}$ morphology, ${ }^{12}$ weighed, diluted 20 -fold in $0.5 \%$ hexadecyl-trimethil-ammonium bromide buffer, and homogenized by bead beating using a tissue homogenizer. Samples were processed and loaded in triplicate into a 96-well plate and exposed to $5 \mathrm{~mm}$ potassium phosphate buffer containing $0.0005 \%$ $\mathrm{H}_{2} \mathrm{O}_{2}$ and $0.167 \mathrm{mg} \mathrm{ml}^{-1} \mathrm{O}$-dianisidine. Data were obtained by time series spectrometry changes in absorbance over $5 \mathrm{~min}$ of incubation at $28^{\circ} \mathrm{C}$. OD changes were automatically recorded every $30 \mathrm{~s}$ to create a kinetic curve from which the steepest slope was used to perform quantifications.

Generation of bone marrow (BM) chimeric mice. Generation of BM chimeric mice was conducted as previously described and depicted in Supplementary Figure S1 online. ${ }^{15}$ Mice receiving BM transfer were irradiated (900 radiation absorbed dose) immediately before transplantation. BM was harvested from femurs and tibias of 10 -wk old SAMP WT or SAMP $\times$ NOD2 ${ }^{-1-}$ donor mice in RPMI medium, and cell suspensions were washed and diluted to a concentration of $30 \times 10^{6}$ cells ml $^{-1}$ of HBSS. A total of $8 \times 10^{6}$ cells $/ 250 \mathrm{ml}$ were injected into the lateral tail vein of recipient mice. BM recipient mice were placed on antibiotic water $(0.7 \mathrm{~mm}$ neomycin sulfate, $80 \mathrm{~mm}$ sulfamethoxazole and $0.37 \mathrm{~mm}$ trimethoprim) for 2 weeks after irradiation and then given autoclaved water for 4 weeks to reconstitute normal gut flora. After 6 weeks, ilea and colons were harvested for fixation (Bouin's solution), SM assessment and histology.

qRT-PCR. SM-micro-dissected samples from ilea and colons were subjected to physical homogenization (with $100 \mathrm{mg}$ of 1.4 -mm ceramic beads, 4,000 r.p.m.), and total RNA was isolated using an RNAeasy Miniprep kit (Qiagen, Valencia, CA, USA). Reverse transcription was performed using the High Capacity RNA-to-cDNA Kit (Applied Biosystems, Carlsbad, CA, USA). qPCR amplification of cDNA samples was performed using clear 96-well plates (Roche, Branchburg, NJ, USA) and the Roche 480 LightCycler SYBR Green (Roche) run template settings (hot start $95^{\circ} \mathrm{C}, 10 \mathrm{~min}, 40$ amplification cycles; $\left.95^{\circ} \mathrm{C}, 15 \mathrm{~s} ; 60^{\circ} \mathrm{C}, 30 \mathrm{~s} ; 72^{\circ} \mathrm{C}, 30 \mathrm{~s}\right) ; 18 \mathrm{~S}$ was used as a reference gene.

Western blot analysis. SM-dissected areas of ilea and isolated MLNs were lysed and centrifuged. Supernatants were standardized through the Bradford assay (BioRad, Hercules, CA), and Western blot was completed on $0.45-\mu \mathrm{m}$ pore-size nitrocellulose membranes (Thermo Scientific, Rockford, IL) and blotted with anti-phopsho-STAT6, antitotal-STAT6, anti-GATA3, anti-IL-13 and anti-actin (AbCam, Cambridge, MA, USA). Immunoreactive bands were revealed with an enhanced chemiluminescent substrate for detection of HRP (Thermo Scientific, Waltham, MA, USA), and blots were exposed to blue basic autorad films (Bioexpress, Kaysville, UT, USA).

Endoscopic investigation. Colonoscopy was performed on day 7 of DSS treatment using a flexible digital ureteroscope (URF-V, Olympus America) under general anesthesia with inhaled isofluorane, USP (Webster Veterinary), as previously described. Inflammation was evaluated using a decimal-scoring system validated for the integrated endoscopic assessment of colonic inflammation and colorectal cancer in mice. $^{35}$

Inter-subject fecal homogenization (IsPreFeH) protocol. Using our previously published IsPreFeH protocol ${ }^{12,19}$ single mice from diverse cages representing both the SAMP WT and SAMP $\times \mathrm{NOD} 2^{-1-}$ colonies were exposed to a composite of fecal sample and pooled bedding material prior to the beginning of the indicated experiments, as an alternative to co-housing. The experimental procedure is illustrated in Supplementary Figure S2.

16S rRNA encoding gene sequencing and microbial community analyses. Community metagenomic DNA was extracted from fecal samples using the PowerSoil-htp 96 Well Soil DNA Isolation Kit (MO BIO Laboratories, Inc., Carlsbad, CA, USA). Amplicons targeting the $\mathrm{V} 4$ region of $16 \mathrm{~S}$ rRNA encoding genes from members of the Bacteria and Archaea using the $515 \mathrm{~F}-806 \mathrm{R}$ primer set $^{36}$ were generated by PCR.

Metatranscriptome sequencing and analyses. Samples were homogenized and stored in RNAlater (Ambion, Foster City, CA, USA) at $-80^{\circ} \mathrm{C}$. RNA was extracted from the re-suspended samples using the MOBIO PowerMicrobiome RNA extraction kit, with a final elution volume of $75 \mu \mathrm{l}$. RNA quality was assessed via RNA integrity number values obtained using Agilent Bioanalyzer software (average RNA integrity number $=6.4 ; n=6$ ). Metatranscriptome libraries were generated following standard protocols in the Argonne NGS core laboratory at the University of Chicago. ${ }^{37}$ Completed metatranscriptome libraries were quality checked on the Agilent Bioanalyzer, quantified using Qubit (Invitrogen, Carlsbad, CA, USA), diluted to $2 \mathrm{~nm}$ and denatured for sequencing on the Illumina MiSeq. Libraries were sequenced 2 per run on a $151 \times 151$ Illumina MiSeq run. Storage and base analyses of the data were provided by the MG-RAST server at Argonne. $^{38}$

Statistics. Statistical analyses were performed using the two-sided unpaired Student's $t$ test or pairwise Mann-Whitney test, with an $\alpha$-level of 0.05 . Logistic regression was conducted using STATA software (v.13 Intercooled, College Station, TX, USA).

Study approval. All experiments were approved by the Institutional Animal Care and Use Committee at Case Western Reserve University and conducted following the Association for Assessment and Accreditation of Laboratory Animal Care guidelines.

SUPPLEMENTARY MATERIAL is linked to the online version of the paper at http://www.nature.com/mi

\section{ACKNOWLEDGMENTS}

F.C. was supported by National Institutes of Health grants DK091222, DK042191, DK055812, DK097948. A.R.P. was supported by a Career Development Award from the Crohn's \& Colitis Foundation of America. The authors thank Mitchell Guanzon, Dennis Gruszka, and Joshua Webster for their technical support. We acknowledge the services of the Mouse Models Core, the Histology/Imaging Core, and the Clinical Component of the $\mathrm{NIH}$ Cleveland Digestive Diseases Research Core Center. 


\section{AUTHOR CONTRIBUTIONS}

D.C., A.R.-P., E.B.C., K.O.A., T.T.P. and F.C. designed experimental studies, analyzed data and wrote the manuscript. D.C., A.R.-P., G.D.S., L.D.M. and D.A.A. conducted experiments, acquired and analyzed data.

\section{DISCLOSURE}

The authors declared no conflict of interest.

(c) 2017 Society for Mucosal Immunology

\section{REFERENCES}

1. Kanneganti, T.D., Lamkanfi, M. \& Nunez, G. Intracellular NOD-like receptors in host defense and disease. Immunity 27, 549-559 (2007).

2. Chen, G., Shaw, M.H., Kim, Y.G. \& Nunez, G. NOD-like receptors: role in innate immunity and inflammatory disease. Annu. Rev. Pathol. 4, 365-398 (2009).

3. Abbott, D.W., Yang, Y., Hutti, J.E., Madhavarapu, S., Kelliher, M.A. \& Cantley, L.C. Coordinated regulation of Toll-like receptor and NOD2 signaling by K63-linked polyubiquitin chains. Mol. Cell Biol. 27, 6012-6025 (2007).

4. Philpott, D.J., Sorbara, M.T., Robertson, S.J., Croitoru, K. \& Girardin, S.E. NOD proteins: regulators of inflammation in health and disease. Nat. Rev. Immunol. 14, 9-23 (2014).

5. Yang, Y., Yin, C., Pandey, A., Abbott, D., Sassetti, C. \& Kelliher, M.A. NOD2 pathway activation by MDP or Mycobacterium tuberculosis infection involves the stable polyubiquitination of Rip2. J. Biol. Chem. 282, 3622336229 (2007).

6. Barrett, J.C. et al. Genome-wide association defines more than 30 distinct susceptibility loci for Crohn's disease. Nat. Genet. 40, 955-962 (2008).

7. Bonen, D.K. et al. Crohn's disease-associated NOD2 variants share a signaling defect in response to lipopolysaccharide and peptidoglycan. Gastroenterology 124, 140-146 (2003).

8. Brant, S.R. et al. Defining complex contributions of NOD2/CARD15 gene mutations, age at onset, and tobacco use on Crohn's disease phenotypes. Inflamm. Bowel Dis. 9, 281-289 (2003).

9. Strober, W. \& Watanabe, T. NOD2, an intracellular innate immune sensor involved in host defense and Crohn's disease. Mucosal Immunol. 4, 484-495 (2011).

10. Corridoni, D., Arseneau, K.O., Cifone, M.G. \& Cominelli, F. The dual role of nod-like receptors in mucosal innate immunity and chronic intestinal inflammation. Front. Immunol. 5, 317 (2014).

11. Pizarro, T.T. et al. SAMP1/YitFc mouse strain: a spontaneous model of Crohn's disease-like ileitis. Inflamm. Bowel Dis. 17, 2566-2584 (2011).

12. Rodriguez-Palacios, A. et al. Stereomicroscopic 3D-pattern profiling of murine and human intestinal inflammation reveals unique structural phenotypes. Nat. Commun. 6, 7577 (2015).

13. Bamias, G. et al. Proinflammatory effects of $\mathrm{TH} 2$ cytokines in a murine model of chronic small intestinal inflammation. Gastroenterology 128, 654-666 (2005).

14. Pietropaoli, D. et al. Occurrence of spontaneous periodontal disease in the SAMP1/YitFc murine model of Crohn disease. J. Periodontol. 85, 1799-1805 (2014).

15. Corridoni, D. et al. Dysregulated NOD2 predisposes SAMP1/YitFc mice to chronic intestinal inflammation. Proc. Natl Acad. Sci. USA 110, 16999-17004 (2013).

16. Gutierrez, O. et al. Induction of Nod2 in myelomonocytic and intestinal epithelial cells via nuclear factor-kappa B activation. J. Biol. Chem. 277, 41701-41705 (2002).

17. Amsen, D., Spilianakis, C.G. \& Flavell, R.A. How are $\mathrm{T}(\mathrm{H}) 1$ and $\mathrm{T}(\mathrm{H}) 2$ effector cells made? Curr. Opin. Immunol. 21, 153-160 (2009).
18. Couturier-Maillard, A. et al. NOD2-mediated dysbiosis predisposes mice to transmissible colitis and colorectal cancer. J. Clin. Invest. 123, 700-711 (2013).

19. Rodriguez-Palacios, A., Aladyshkina, N. \& Cominelli, F. Stereomicroscopy and 3D-target myeloperoxidase intestinal phenotyping following a fecal flora homogenization protocol. Protoc. Exchange; doi:10.1038/protex.2015.065 (2015).

20. Negroni, A. et al. Activation of NOD2-mediated intestinal pathway in a pediatric population with Crohn's disease. Inflamm. Bowel Dis. 15, 1145-1154 (2009).

21. Jamontt, J., Petit, S., Clark, N., Parkinson, S.J. \& Smith, P. Nucleotidebinding oligomerization domain 2 signaling promotes hyperresponsive macrophages and colitis in IL-10-deficient mice. J. Immunol. 190, 2948-2958 (2013).

22. Bamias, G., Corridoni, D., Pizarro, T.T. \& Cominelli, F. New insights into the dichotomous role of innate cytokines in gut homeostasis and inflammation. Cytokine 59, 451-459 (2012).

23. Mannon, P. \& Reinisch, W. Interleukin 13 and its role in gut defence and inflammation. Gut 61, 1765-1773 (2012).

24. Allen, J.E., Sutherland, T.E. \& Ruckerl, D. IL-17 and neutrophils: unexpected players in the type 2 immune response. Curr. Opin. Immunol. 34, 99-106 (2015).

25. Hogan, S.P., Waddell, A. \& Fulkerson, P.C. Eosinophils in infection and intestinal immunity. Curr. Opin. Gastroenterol. 29, 7-14 (2013).

26. Fournier, B.M. \& Parkos, C.A. The role of neutrophils during intestinal inflammation. Mucosal Immunol. 5, 354-366 (2012).

27. Magalhaes, J.G. et al. Nucleotide oligomerization domain-containing proteins instruct T cell helper type 2 immunity through stromal activation. Proc. Natl Acad. Sci. USA 108, 14896-14901 (2011).

28. Bamias, G. et al. Commensal bacteria exacerbate intestinal inflammation but are not essential for the development of murine ileitis. J. Immunol. 178, 1809-1818 (2007).

29. Petnicki-Ocwieja, T. et al. Nod2 is required for the regulation of commensal microbiota in the intestine. Proc. Natl Acad. Sci. USA 106, 15813-15818 (2009).

30. Ramanan, D., Tang, M.S., Bowcutt, R., Loke, P. \& Cadwell, K. Bacterial sensor Nod2 prevents inflammation of the small intestine by restricting the expansion of the commensal Bacteroides vulgatus. Immunity 41, 311-324 (2014).

31. Robertson, S.J. et al. Nod1 and Nod2 signaling does not alter the composition of intestinal bacterial communities at homeostasis. Gut Microbes 4, 222-231 (2013).

32. Bielig, $\mathrm{H}$. et al. Anti-inflammatory arene-chromium complexes acting as specific inhibitors of NOD2 signalling. ChemMedChem 5, 2065-2071 (2010).

33. Corridoni, D., Arseneau, K.O. \& Cominelli, F. Inflammatory bowel disease. Immunol. Lett. 161, 231-235 (2014).

34. Tigno-Aranjuez, J.T. et al. In vivo inhibition of RIPK2 kinase alleviates inflammatory disease. J. Biol. Chem. 289, 2965129664 (2014).

35. Kodani, T. et al. Flexible colonoscopy in mice to evaluate the severity of colitis and colorectal tumors using a validated endoscopic scoring system. J. Vis. Exp. 16, e50843 (2013).

36. Caporaso, J.G. et al. Global patterns of $16 \mathrm{~S}$ rRNA diversity at a depth of millions of sequences per sample. Proc. Natl Acad. Sci. USA 108, 4516-4522 (2011).

37. Hampton-Marcell, J.T., Moormann, S.M., Owens, S.M. \& Gilbert, J.A. Preparation and metatranscriptomic analyses of host-microbe systems. Methods Enzymol. 531, 169-185 (2013).

38. Meyer, F. et al. The metagenomics RAST server-a public resource for the automatic phylogenetic and functional analysis of metagenomes. BMC Bioinf. 9, 386 (2008). 